\title{
Boundedness and Continuity Implications on a Solution of an Initial Value Problem of First Order Linear Differential Equation
}

\author{
${ }^{1}$ Eze, Everestus Obinwanne, ${ }^{2}$ Egbuhuzor, Udechukwu B. \\ ${ }^{I}$ Department of Mathematics,Michael Okpara University of Agriculture, Umudike-Abia state Nigeria. \\ ${ }^{2}$ Department of Mathematics/Statistics,Caritas University, Amorji-Nike, Enugu state Nigeria.
}

\begin{abstract}
The theories of solutions of Differential Equations are way important because of their applicability to areas of physical science and modeling of some physical phenomena. The notion of their properties are quite revealing because of the cyclic relationship existing between them. In this paper, our objective is to x-ray the boundedness and continuity implications in a solution of an initial value problem of a first-order linear differential equation. We employed Arzela-Ascoli theorem and some other theorems from bounded linear maps to prove our assertions. The results obtained were quite revealing and shows the existence of a local solution to our equation if only $f$ is assumed to be continuous. Furthermore, that map continuity is equivalent to boundedness in Normed linear spaces. We concluded that our objectives were achieved based on our deductions.
\end{abstract}

Keywords: Boundedness, Continuity, Arzela-Ascoli Theorem, Normed linear map

\section{Introduction}

There are many qualitative properties of solutions of any differential equations, for instance, continuity, Boundedness, Periodicity, Uniform convergence, Phase plane, Existence and uniqueness etc. in all these properties, or finds out there are relationships among these properties leading to the solutions of any Differential Equation. For instance, we find out that Boundedness which is one of the properties of uniform convergence implies continuity and continuity implies continuity at the origin and continuity at the origin implies Lipschitz continuous and Lipschitz continuous implies Boundedness(Note that these can occur in bounded linear maps) C. E. Chidume(2006). If we drop that $\mathrm{f}$ is Lipschitz we still have existence of solutions but we already know that we might lose uniqueness. Even without uniqueness, two given solutions of IVP can still be glued together at to (if necessary) to obtain a solution defined on $I_{1} U I_{2}$. Furthermore, Zonis lemma can be used to ensure existence of maximal solutions in this case. [Gerald Teschl, 2012]

It is well known that "compactness" is central in Mathematical Analysis and its numerous applications. In fact, one characterization of finite dimensional spaces is a classical result known as the Heine-Borel theorem which states that "a normed linear space $\mathrm{E}$ is finite dimensional if and only if the unit ball in $\mathrm{E}$ is compact. Another important classical result connected with compactness is the well known Bolzano-Weierstrass theorem which states that any bounded sequence in $\mathrm{R}$ has a convergent subsequence. [C. E. Chidume, 2006]

To show that the continuity of $\mathrm{f}$ is sufficient for the existence of at least the solution of our equation (1.1), in the proof of Euler's method, the key observation is that, since $\mathrm{f}$ is continuous, it is bounded by a constant on each compact interval. Hence the derivative of $x_{h}(t)$ is bounded by the same constant. Since then constant is independent of $\mathrm{h}$, the functions $x_{h}(t)$ form an equi-continuous family of functions which converges uniformly after may be passing to a subsequence by the Arzela theorem. [Gerald Teschl, 2012]

In this paper, our objective is to search for the boundedness and continuity implications in a solution of an initial value problem of a first order differential Equation. We will invoke particularly Arzela-Ascoli theorem to prove our case. So many authors have proved the existence of solutions of the Differential Equations by using Boundedness properties,[Finan M.B(1999), Ogbu et al(2012)]. Others have also used uniform convergence [Eze E.O et al(2012, 2013)]

In recent years, there has been an interest in the study of fractional integro-fractional differential equations. S. Momani (2000) used Schauder's fixed point theorem to obtain local existence and Tychonov's fixed point theorem to obtain global existence of solutions of (1.1). S. Momani (2001) used the successive approximations method and Arezela-Ascoli lemma to obtain existence and uniqueness of solutions of equation (1.1). S. Momani and R. EL-Khazali (2000) using comparison principle and Ascoli lemma investigated the existence of extremal (maximal and minimal) solutions of our equations (1.1). S. Momani et al (2007) used Bihari's inequality to obtain local uniqueness and Gronwall's inequality to obtain global uniqueness of solution of the initial value problem (1.1) which they considered as a generalization of the earlier work on S. M. Momani (2000).

The requirement that $\mathrm{f}$ is locally Lipschitz continuous in Picard-Lindelof theorem is already more than we actually needed in its proof. In fact, all one needs to require is that $\mathrm{f}$ is measurable with $M(t)$ finite and $L(t)$ 
locally integrable (ie $\int L(t) d t<\infty$ for any compact interval I). However, then solution of the integral equation is only absolutely continuous and might fail to be continuously differentiable. In particular when going back from integral to the differential equation, the differentiation has to be understood in a generalized sense. [Gerald Teschl, 2012]

In the case of continuity, one notices that although a function might be continuous, it might not be differentiable but all differentiable functions are continuous. This is because the domains of differentiable functions are larger than the domain of continuous functions. [Osisiogu U.A. (2009)]

$$
\begin{aligned}
& \text { We consider the first order differential equation of the form. } \\
& \left.\begin{array}{l}
\dot{x}=f(t, x) \\
x\left(t_{0}\right)=x_{0}
\end{array}\right\}
\end{aligned}
$$

\section{Definitions And Theorems}

We will state some definitions and theorems which we consider as an asset to our proof.

Definition 2.1: Let $X$ and $Y$ be normed linear spaces and let $T: X \rightarrow Y$ be a linear map. Then $\mathrm{T}$ is said to be bounded if there exists some constant $k \geq 0$ such that for each $x \in X$

$\|T(x)\| \leq k\|x\|$,

The constant $k$ is called a bound for $T$ and in this case T is called a bounded linear map [C.E Chidume(2006)]

Definition 2.2: Let $(X, P X)$ and $(Y, P y)$ be metric spaces and let

$$
f: D(f) \in X \rightarrow Y
$$

be a mapping from the domain of $f, D(f)$ in $X$ to $Y$. Then $f$ is said to be continuous at $x_{0} \in X$ if given any $\varepsilon>0$, there exists $\delta>0$ such that if $x \in D(f)$ and $P x\left(x, x_{0}\right)<\delta$ then $P y\left(x, x_{0}\right)<\varepsilon$ [C.E Chidume(2007)]pp 74 .

Theorem 2.3: Let $X$ and $Y$ be normed linear spaces and let $T: X \rightarrow Y$ be a linear map. Then the following statements are equivalent

i. $\quad T$ is continuous

ii. $\quad T$ is continuous at the origin (in the sense that if $\left\{x_{n}\right\}$ is a sequence in $X$ such that $x_{n} \rightarrow 0$ as $n \rightarrow$ $\infty$, then $T x_{n} \rightarrow 0$ in $\left.Y n \rightarrow \infty\right)$

iii. $\quad T$ is Lipschitz. i.e, there exists a constant $k \geq 0$ such that $x \in X$

$$
\|T x\| \leq k\|x\|
$$

iv. If $D:=\{x \in X:\|x\| \leq 1$ is a closed unit disc in $X\}$; then $T(D)$ is bounded (in the sense that there exist a constant $M \geq 0$ such that

$\|T x\| \leq M$ for all $x \in D$

Proof: (see Chidume C.E. 2006) pp. 25

Remark: In the light of definition 2.1 and theorem 2.3, we have that a linear map $T: X \rightarrow Y$ is continuous if and only if it is bounded. Thus for linear maps, continuity and boundedness are equivalent.

Theorem 2.4(Arzela- Ascoli Theorem): If $X$ is a compact metric space, a subset $K \subset C(X)$ is relatively compact if and only if it is uniformly bounded and equi-continuous.

Theorem 2.5(Bolzano Weirstress theorem): Every bounded sequence in $\Re$ has a convergent subsequence.

We state the Existence and Uniqueness theorem for equation (1.1) as follows[C.E. Chidume; 2003 et al].

Theorem 2.6(PICARDS): Let $\Re$ be a region in the $(t, x)$ plane containing the point $\left(t_{o}, x_{o}\right)$ and suppose that;

i. $\quad f$ is a continuous function in the interval $I$

ii. $\quad f$ is lipschitz continuous with lipschitz constant $K$, then the initial value problem

has a unique solution in the closed interval

$$
\dot{x}=f(t, x) ; x\left(t_{o}\right)=x_{o}
$$

Proof: See Ogbu et al (2012), Finan M.B(1999)

$$
I=\left[t_{o}-h, t_{o}+h\right]
$$

Theorem 2.7(PICARDS): The Existence and Uniqueness theorem can be re-stated as follows; if $f(t, x)$ and $\frac{\partial f}{\partial x}$ are continuous for all $(t, x)$ in the rectangular plane $\Re$ and bounded that is;

$$
\begin{array}{ll}
\text { i. } & |f| \leq K \\
\text { ii. } & \left|\frac{\partial f}{\partial x}\right| \leq M
\end{array}
$$

for all $(t, x)$ in $\Re$, the initial value problem (1.1) has at most one solution $x(t)$. Hence it has precisely one solution. See Ogbu et al (2012), Finan M.B (1999).

One of the approaches to approximate a solution (1.1) is by constructing a sequence of functions that converges uniformly to a solution. This approach is due to Picard. Picard's iterations are defined recursively as 
$x\left(\mathrm{t}_{\mathrm{o}}\right)=x_{0}$

$$
\begin{aligned}
& x_{1}\left(t_{1}\right)=x_{o}+\int_{t_{o}}^{t} f\left(S, x_{0}(s)\right) d s \\
& x_{2}\left(t_{2}\right)=x o+\int_{t_{o}}^{t} f\left(S, x_{1}(s)\right) d s \\
& \cdot \\
& \cdot \\
& x_{n}\left(t_{n}\right)=x_{o}+\int_{t_{o}}^{t} f\left(S, x_{n-1}(s)\right) d s
\end{aligned}
$$

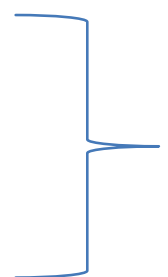

If this sequence of functions of (1.2) converges uniformly to a function $x(t)$ then the function is the solution to our initial value problem (1.1). We have established this in the proof of the theorems(Eze E.O (2012) Ogbu et al (2012), Finan M.B (1999).

\section{Methodology}

We will use the Arzela- Ascoli's theorem to prove the relationship between boundedness and continuity. Furthermore, we will employ the theorem in linear maps to show that equivalence between continuity and boundedness and draw conclusions based on their implications to the solution of our initial value problem of a first order differential equation of the form

$$
\left.\begin{array}{l}
\dot{x}=f(t, x) \\
x\left(t_{0}\right)=x_{0}
\end{array}\right\}
$$

\section{Main Results}

We will use the proof of Arzela-Ascoli theorem to seek for equi-continuity and uniform convergence (Theorem 2.4). Again, we will show that boundedness implies continuity. An application of the Arzela-Ascoli Theorem yields the existence of solutions to the differential equation of (1.1) or $y^{\prime}=f(x, y)$ in case $f$ is assumed to be continuous.

Theorem: If $f$ is continuous on an open set then, every $\left(t_{o}, x_{o}\right) \in D$, the differential equation $\frac{d y}{d u}=$ $f(t, x)$ has a local solution passing through $\left(t_{o}, x_{o}\right)$

Proof: Let $\Re$ be a closed, non-degenerate rectangle contained in D having centre $\left(t_{o}, x_{o}\right)$ and sides parallel to the axes. Let $M>0$ be such that $|f(t, x)|<M$ for all $(t, x) \in \Re$

The two lines through $\left(t_{o}, x_{o}\right)$ with slopes $I M$ may intersect the horizontal sides of $\Re$. If so, take $I$ to be the projection on the $\mathrm{x}$-axis of the interval between the points of intersection. Otherwise, $I$ is the projection of the entire horizontal side. In either case, I has the form $\left[t_{o}-a, t_{o}+a\right]$ for some $a>0$. Our solution X will be defined on I. It will be obtained by applying the Arzela-Ascoli theorem in C(I) to a set of "approximate" solutions.

For $\varepsilon>0$ we shall call a function $\varphi_{\epsilon}$ defined on I an $\varepsilon$-approximate solution if its graph is a polynomial arc through $\left(t_{o}, x_{o}\right)$ lying between the two lines of slope $\pm M$ and the lines $t=t_{o}+a$ and satisfying $\left|\dot{X}(t)-f\left(t, \varphi_{\epsilon}(t)\right)\right|<\varepsilon$, at each $x \in I$, where $\dot{X}_{\epsilon}$ exists (i.e except at the finite number of points where the vertices occur. Since $f$ is uniformly continuous on $\Re$, there is a $\delta>0$ such that for $(t, x)$ and $(\bar{t}, \bar{x})$ in $\Re$

$|t-\bar{t}|<\delta$ and $|x-\bar{x}|<\delta$ imply $|f(t, x)-f(\bar{t}-\bar{x})|<\varepsilon$

It is enough to describe $\varphi_{\epsilon}$ on the interval $\left[t_{o}, t_{o}+a\right]$

Divide this interval by points $t_{o}<t_{1}<t_{2}<\ldots<t_{n}=t_{o}+a$

So that

$$
\left|t_{i}-t_{i-1}\right|<\min \left(\delta, \frac{\delta}{M}\right) i=1, \ldots, n
$$

Let $\varphi_{\epsilon}\left(t_{o}\right)=x_{o}$ and in $\left[t_{o}, t_{1}\right]$. Let $\varphi_{\epsilon}$ be linear with slope $f\left(t_{1}, \varphi_{\epsilon}\left(t_{1}\right)\right)$. Continuing in a way which should now be clear, $\varphi_{\epsilon}$ is defined on $\left[t_{o}, t_{o}+a\right]$ since the slope of $\varphi_{\epsilon}$ nowhere exceeds $\mathrm{M}$ in absolute value, we obtain $\left|\varphi_{\epsilon}(\dot{t})-\varphi_{\epsilon}(\bar{t})\right| \leq M|t-\bar{t}|$ for all $t, \bar{t} \in I$

In other words, the functions $\varphi_{\epsilon}$, where we consider one such function for each $0<\varepsilon<a$ are an equicontinuous collection

Fix $\epsilon$. If $x \in$ Iand $t \neq t_{i}, i=0,1, \ldots, n$ then $t_{j-1}<t<t_{j}$ for some $j$ and

But this implies

$$
\left|\varphi_{\epsilon}(t)-\varphi_{\epsilon}\left(t_{j-1}\right)\right| \leq M\left|t-t_{j-1}\right|<M \cdot \frac{\delta}{M}=\delta
$$

$$
\mid f\left(t_{j-1}\right), \varphi_{\epsilon}\left(t_{j-1}\right)-f\left(t, \varphi_{\epsilon}(t) \mid<\varepsilon\right.
$$

But $\varphi_{\epsilon}^{\prime}(t)$ exists and equals $f\left(x_{j-1}, \varphi^{\prime}\left(t_{j-1}\right)\right)$. It follows that $\varphi_{\epsilon}$ is an $\varepsilon-$ appropriate solution.

$$
\mid \varphi_{\epsilon}^{\prime}(t)-f\left(t, \varphi_{\epsilon}(t) \mid<\varepsilon, t \in I \text { and } t \neq t_{i,},=1, \ldots, n\right.
$$


We now write

$$
\varphi_{\epsilon}(t)=x_{o}+\int_{t_{o}}^{t}\left\{f \left(s, \varphi_{\epsilon}(s)+\left[\varphi_{\epsilon}^{\prime}(t)-f\left(t, \varphi_{\epsilon}(t)\right]\right\}\right.\right.
$$

Where $\varphi_{\epsilon}^{\prime}$ can have any values at the vertices. Choose them so that the term in brackets vanishes there.

The collection $\varphi_{\epsilon}, a>\varepsilon>0$ is uniformly bounded on I (since graphs all lie in $\mathfrak{R}$ ) and is equicontinuous. Hence if $\left(\varepsilon_{n}\right)$ is a null sequence, it has a subsequence, which we again call $\left(\varepsilon_{n}\right)$ such that $\left\{\varphi_{\epsilon_{n}}\right\}$ converges uniformly to a function $Q$ on $I$.

Since $f$ is uniformly continuous $\left\{f(t), \varphi_{\epsilon_{n}}(t)\right\}$ converges uniformly to $f(s, \varphi(s)$ on $I$. Moreover, $\varphi_{\epsilon_{n}}^{\prime}(t)-f(u, \varphi(u))$ converges uniformly to zero on I. it then follows by $\left.{ }^{*}\right)$ that

$$
X(t)=x_{o}+\int_{t_{o}}^{t} f\left(t_{s}, X(t)\right) d s
$$

But this implies that $\varphi$ is differentiable on $I$ and is the required solution [Casper G and George P.(1987)].

Evoking Theorem (2.3) and we offer the proof as follows:

Boundedness $\Rightarrow$ continuity

Proof $\Rightarrow$ Let $x_{1}, x_{2}$ be arbitrary elements of $\mathrm{X}$. assume that $x_{1}-x_{2} \neq 0$. Consider the vector:

$$
U: \frac{x_{1}-x_{2}}{\left\|x_{1}-x_{2}\right\|}
$$

Clearly; $u \in D$ and so by condition (iv) (i.e. T(D) is bounded) there exists a constant $k \geq 0$ such that;

$\|T(u)\| \leq K$, i.e.

$$
\left\|T\left(\frac{x_{1}-x_{2}}{\left\|x_{1}-x_{2}\right\|}\right)\right\| \leq K \text { or }\left\|T x_{1}-T x_{2}\right\| \leq K\left\|x_{1}-x_{2}\right\|
$$

$\Rightarrow$ If on the other hand, $x_{1}-x_{2}=0$ this inequality clearly also holds. Thus, the inequality holds for all $x_{1}, x_{2} \in$ $X$. Now given any $\varepsilon>0$.

Choose $\delta=\frac{\varepsilon}{k+1}$; so if $\left\|x_{1}-x_{2}\right\|<\delta$ we obtain

$$
\left\|T x_{1}-T x_{2}\right\| \leq K\left\|x_{1}-x_{2}\right\| \leq K \delta=\left(\frac{K}{K+1}\right) \varepsilon<\varepsilon
$$

And hence $T$ is uniformly continuous on $X$ [C.E. Chidume;2006].

\section{Discussion}

We look at this theorem: Let $\left\{f_{n}\right\}$ be a sequence of continuous function on a set D. Let $f_{n} \rightarrow f$ uniformly on $D$. Then $f$ is continuous on $D$. This theorem is useful in some cases, in showing that a given sequence of continuous functions does not converge uniformly. The theorem asserts the following:

i. $\quad\left\{f_{n}\right\}$ is a sequence of continuous function; $f_{n} \rightarrow f$ uniformly $\Rightarrow f$ is continuous. The contrapositive statements to this theorem(i.e, another way of stating the same theorem) is the following:

$\left\{f_{n}\right\}$ is a sequence of continuous function; $f_{n} \rightarrow f$ pointwise, then $f$ is not continuous

$\Rightarrow$ convergence is not uniform.(C.E. Chidume et al 2003)

Arzela-Ascoli propagation theorem asserts that pointwise convergence of an equi-continuous sequence of functions on a dence subset of the domain propagates to uniform convergence on the whole domain (C.E. Chidume et al 2003).

The Weierstrass theorem asserts that if $[a, b]$ is a closed interval on the real line, then the polynomials are dense in $c[a, b]$. In other words, every continuous function on $[a, b]$ is the limit of a uniformly convergent sequence of polynomials [Gasper Goffman and George Patrick(1987)].

$T$ is continuous if and only if for every open $G \subset Y$ the inverse image $T^{-1}(G)=\left\{x: x \in X_{1}, T_{x} \in G\right\}$ is open in $X$.

Every totally bounded metric space is separable.

$T$ is said to be uniformly continuous on $X$ if for every $\varepsilon>0$ there is a $\delta>0$ such that $p\left(x x^{\prime}\right)<\delta \Rightarrow \delta\left(T_{x}, T_{x}^{\prime}\right)<\varepsilon$ for all $x, x^{\prime} \in X$

If $X$ is compact then, every T which is continuous on $X$ is uniformly continuous.

A collection $F$ of function on a set $X$ is said to be uniformly bounded if there exists $M>0$ such that $|f(x)| \leq M$ for all $x \in X$ and all $f \in F$.

Uniform convergence implies semi-uniform convergence implies point-wise convergence implies $L^{2}$ space (continuous and bounded function) but the converse is not true. [Eze, E.O. et al, 2013]

Asymptotic stability implies stability implies Boundedness implies periodicity. [Eze, E.O. et al, 2013]

All uniform convergence solutions are equally bounded solution but the converse may not be true. 


\section{Conclusion}

If a sequence of function $x_{n}(t)$ converges uniformly to the solution $x(t)$ of our initial value problem then there must also exist a subsequence $\left\{x_{n_{k}}\right\}$ of any bounded equi-continuous sequence of function $\left\{x_{n}\right\}$ which must converge uniformly to our $x(t)$. This point $x(t)$ is a unique point which coincides with our unique solution of (1.1) and therefore confirms existence of solution (1.1). Again, an affirmation of our theorem on existence and uniqueness of solution of (1.1) due to Picard's theorem and re-emphasis on boundedness and continuity of our function $f$.

\section{References}

[1] Casper Goffman and George Pedrick (1987): First Course in Functional Analysis, Prentice-Hall of India Private Limited New-Delhi

[2] C. E. Chidume (2003): Foundations of Riemann Integration. International Centre for Theoretical Physics, Trieste, Italy

[3] C. E. Chidume (2006): Applicable Functional Analysis. International Centre for Theoretical Physics, Trieste, Italy

[4] Eze E.O, Ogbu H.M and Aja O.R (2013): On Application of Lyapunov and Yoshizawa's Theorems to Stability, Asymptotic Stability Boundedness and Periodicity of Solutions of Duffing's Equation. Book of Abstracts of the Nigerian Mathematical Society Conference Ile-Ife

[5] Eze E.O, Ogbu H.M and Aja O.R (2013): On the Uniform Convergence to the Solution of the Intial Value Problem of the NthOrder Differential Equation. Journal of the Nigerian Mathematical Physics

[6] Eze E.O, Ugbene I.J. And Ogbu H.M. (2012) On Demand for Uniqueness of Solutions of Initial Value Problem of a First Order Differential System: Icastor Journal of Mathematical Sciences, India Vol 6 No2

[7] Eze.E.O, Ugbene Jeff and Ogbu. H.M. [2013] On Uniform Convergence to a Solution of Initial Value Problem of a First Order Linear Differential System.IcastorJournal of Mathematical Science India Vol 7 No 1

[8] Finan, M. B. (1999): A First Course in Elementary Differential Equations; Arkansas Tech. University Press

[9] Gerald Teschl (2012): Ordinary Differential Equations and Dynamical Systems Published by American Mathematical Society, Niema Austria

[10] Ogbu H.M. and Eze E.O. (2012). An Insight on Existence and Uniqueness of Solutions for an Initial Value Problem of First Order Differential Equation, World Journal Series. Accepted for publication with receipt on payment

[11] Osisiogu U.A (2009): A First Course Mathematical Analysis and Differential Equations, Best Soft Educational Books; Festal Town, Lagos, Nigeria

[12] Qiuyi Dai and Christopher C. Tisdell, (2006): Existence of Solutions for First Order Dynamic Boundary Value Problems. International Journal of Difference Equations Vol. 1 No. 1 pp 1-17, Research India Publications

[13] S. Momani (2000): Local and Global Existence Theorems on Fractional Integro-Differential Equations, Journal of Fractional Calculus Vol. 18 pp 81-86

[14] S. Momani (2001): Some Existence Theorems on Fractional Integro-Differential Equations, Abhath-AL Yarmouk Journal 10(26) pp 435-444

[15] S. M. Momani and R. El-Khazali (2000): On the Existence of Extremal Solutions of the Fractional Integro-Differential Equations, Journal of Fractional Calculus Vol. 18 pp 87-92

[16] Shaher Momani, Ahlam Jameel and S. Al-Azawi (2007): Local and Global Theorems on Fractional Integro-Differential Equations via Bihar's and Gronwalls inequalities. Soochow Journal of Mathematics Vol. 33 No. 4 pp 619-627 